\title{
Potencialidades e desafios para a educação interprofissional no contexto da
}

\section{graduação em cursos da saúde}

Opportunities and barriers for interprofessional education in the context of undergraduate health

\author{
courses
}

Potencialidades y desafíos de la educación interprofesional en el contexto de los cursos de pregrado en salud

Luis Rocildo Caracas Vieira e Souza ORCID: https://orcid.org/0000-0002-9739-7993 Universidade Estadual do Ceará, Brasil E-mail: rocildo.nutri@gmail.com

Maria Marlene Marques Ávila ORCID: https://orcid.org/0000-0002-8511-2524 Universidade Estadual do Ceará, Brasil E-mail: marlene.avila@uece.br

\begin{abstract}
Resumo
A Educação Interprofissional (EIP) representa uma alternativa de reorientação da formação profissional no contexto do Sistema Único de Saúde (SUS). Este estudo avaliou a implantação de uma disciplina interprofissional em uma universidade pública cearense, por meio do referencial teórico-metodológico da Avaliação de Quarta Geração. Foram informantes 22 discentes e 13 docentes. As informações foram construídas por meio da observação e de entrevistas semiestruturadas virtuais, dada a circunstância da pandemia de Covid-19. A validação das informações e delimitação dos marcadores avaliativos foi realizada por meio de oficinas de negociação com os grupos de interesse. Abordamos os marcadores avaliativos de duas categorias temáticas: as potencialidades e os desafios para a disciplina de EIP. Em relação às potencialidades, discutimos a oportunidade de aprendizado compartilhado e interativo, a valorização do SUS e a ênfase no trabalho em equipe e colaboração. Na categoria desafios, analisamos a não adesão de todos os cursos de saúde da instituição, as distintas modalidades de oferta da disciplina e a necessidade de desenvolvimento docente para a EIP. O estudo demonstrou a potência do aprendizado compartilhado e interativo entre estudantes de diferentes cursos de saúde, e a necessidade de um maior apoio institucional para a ampliação e qualificação da educação interprofissional.
\end{abstract}

Palavras-chave: Educação interprofissional; Educação baseada em competências; Saúde coletiva; Educação superior; Ensino.

\begin{abstract}
The Interprofessional Education (IPE) represents an alternative for reorienting professional training in the context of the Brazilian Unified Health System (SUS). This study evaluated the implementation of an interprofessional discipline in a public university in Ceará, through the theoretical-methodological framework of Fourth Generation Evaluation. 22 students and 13 professors were informants. The information was built through observation and virtual semistructured interviews, given the circumstance of the Covid-19 pandemic. Validation of information and delimitation of evaluative markers was carried out through negotiation workshops with stakeholders. We approach the evaluative markers of two thematic categories: the opportunities and barriers for the IPE discipline. Regarding the opportunities, we discussed the opportunity for shared and interactive learning, the appreciation of the SUS and the emphasis on teamwork and collaboration. In the barriers category, we analyzed the non-adherence of all health courses in the institution, the different modalities of offering the discipline and the need for professors development for the IPE. The study demonstrated the power of shared and interactive learning among students from different health courses, and the need for greater institutional support for the expansion and qualification of IPE.
\end{abstract}

Keywords: Interprofessional education; Competency-based education; Public health; Higher education; Teaching.

\section{Resumen}

La Educación Interprofesional (EIP) representa una alternativa para reorientar la formación profesional en el contexto del Sistema Único de Salud (SUS). Este estudio evaluó la implementación de una disciplina interprofesional en una universidad pública de Ceará, a través del marco teórico-metodológico de la Evaluación de Cuarta Generación. 22 estudiantes y 13 profesores fueron informantes. La información se construyó a través de la observación y entrevistas 
semiestructuradas virtuales, dada la circunstancia de la pandemia Covid-19. La validación de información y delimitación de marcadores evaluativos se realizó mediante talleres de negociación con grupos de interés. Abordamos los marcadores evaluativos de dos categorías temáticas: los potenciales y desafíos para la disciplina EIP. En cuanto al potencial, discutimos la oportunidad de aprendizaje compartido e interactivo, la valoración del SUS y el énfasis en el trabajo en equipo y la colaboración. En la categoría desafíos, se analizó la no adherencia de todos los cursos de salud de la institución, las diferentes modalidades de oferta de la disciplina y la necesidad de desarrollo docente para la EIP. El estudio demostró el poder del aprendizaje compartido e interactivo entre estudiantes de diferentes cursos de salud y la necesidad de un mayor apoyo institucional para la expansión y calificación de la educación interprofesional.

Palabras clave: Educación interprofesional; Educación basada en competencias; Salud pública; Educación superior; Enseñanza.

\section{Introdução}

Os debates a respeito da formação em saúde no ensino superior tomaram ênfase nas últimas décadas, muito em razão da nova conjuntura nas políticas de saúde após a implantação e regulamentação do Sistema Único de Saúde (SUS) e seus princípios doutrinários de universalidade, integralidade e equidade, além de seu papel legal de ordenador da formação de recursos humanos na saúde. Considerando o imperativo da concretização desses princípios e o contexto da crescente complexidade e dinamicidade das demandas de saúde da população, há a necessidade de profissionais aptos para o desenvolvimento do trabalho em equipe e da prática colaborativa (Lei no 8.080, 1990; Ely \& Toassi, 2018).

Contudo, a formação de profissionais de saúde no ensino superior apresenta complexos desafios históricos, institucionais e curriculares quando se considera o processo social e político de construção do SUS e as demandas de saúde da população brasileira. $\mathrm{O}$ atual modelo formativo hegemônico uniprofissional, disciplinar e com ênfase nos saberes específicos da profissão, contribui para uma forte divisão do trabalho, o tribalismo profissional e o desenvolvimento de práticas profissionais fragmentadas, acarretando prejuízos para a assistência dispensada aos usuários dos serviços (Costa, 2017; Costa, Peduzzi, et al., 2018; Freire Filho et al., 2018).

Como resposta aos desafios da formação em saúde e alternativa à formação estritamente uniprofissional, a educação interprofissional (EIP) tem ganhado destaque no Brasil e no mundo. A EIP tem como objetivo a formação de profissionais mais aptos para o trabalho em equipe colaborativo, resultando em benefícios como a ampliação da capacidade resolutiva dos sistemas de saúde, a redução de erros no cuidado, uma maior satisfação de usuários após tratamento ofertado por uma equipe colaborativa, assim como a redução de conflitos e tensões entre profissionais (Barr, 2015; Capozollo, 2017; Frenk et al., 2010; Girard, 2021; Organização Mundial da Saúde [OMS], 2010; Stadick, 2020; Toassi et al., 2020). As iniciativas de EIP promovem momentos de aprendizado compartilhado e interativo entre estudantes de diferentes cursos para o desenvolvimento de competências necessárias ao trabalho coletivo (Ceccim, 2017; Reeves, 2016). Não se trata apenas de juntar estudantes em um mesmo espaço físico. O aprendizado conjunto deve ser permeado pela comunicação, onde os participantes aprendem com, para e entre si, mobilizando diferentes saberes e práticas para romper com a fragmentação do conhecimento e das práticas profissionais (Ceccim, 2018; Ellery, 2018; OMS, 2010).

No contexto da formação em saúde, as recomendações atuais direcionam à preparação de profissionais com perfil generalista, humanista, crítico e reflexivo, assimilando valores, habilidades e práticas necessárias para o trabalho em equipe resolutivo. Assim, as Instituições de Ensino Superior (IES) assumem relevância na formação em saúde ao inserir a EIP nos desenhos curriculares dos cursos de saúde (Ceccim, 2018; Ellery, 2018; Peduzzi \& Agreli, 2018).

A Universidade Estadual do Ceará (UECE) tem caminhado na direção de inserir a EIP nos currículos de saúde ao ofertar a disciplina Introdução à Formação Interprofissional para o Sistema Único de Saúde (IFISUS) no primeiro semestre dos cursos de Ciências Biológicas, Medicina, Nutrição e Terapia Ocupacional, com alunos mesclados em turmas interprofissionais. Experiência pioneira na instituição, a disciplina foi planejada e implantada pelo Centro de Ciências da Saúde (CCS) no âmbito do Programa de Reorientação da Formação dos Profissionais de Saúde (Pró-Saúde) abrangendo os cursos supracitados. 
Importante ressaltar que também integram o CCS os cursos de Enfermagem e Educação Física e em outros Centros de Estudos da IES existem os cursos de Serviço Social, Psicologia e Medicina Veterinária.

Embora a IFISUS represente uma evidente inovação curricular que articula a EIP com a formação em saúde, é preciso ressaltar que se constitui como uma disciplina em fase inicial de desenvolvimento, uma vez que a sua primeira oferta ocorreu em 2019. Nesse caso, é fundamental avaliar sua implantação, as potencialidades e os desafios relacionados com o desenvolvimento da IFISUS, o que possibilita contribuir com a qualificação da experiência de EIP na IES, bem como conhecer melhor possíveis aspectos relacionados à implantação da EIP na universidade pública brasileira.

Apesar do reconhecimento dos benefícios advindos da EIP para a formação dos profissionais de saúde, ainda são poucas as experiências - muitas vezes isoladas - de IES que a desenvolvem, demandando mais pesquisas sobre os resultados dessas inciativas no ensino superior na saúde (Rossit, Freitas, et al., 2018; Rossit, Santos Junior, et al., 2018).

A literatura evidencia a necessidade de investigações sobre as experiências de EIP para estudantes da saúde e seus impactos na formação de profissionais alinhados com os princípios e diretrizes do SUS (Costa, Peduzzi, et al., 2018; Mello et al., 2014; Moraes, 2016).

Considerando a relevância da temática da formação em saúde e o crescente interesse e investimentos na EIP, foi desenvolvida a pesquisa Formação Interprofissional em Saúde: avaliação da experiência na Universidade Estadual do Ceará, da qual esse estudo constitui um recorte que aborda o objetivo de identificar as potencialidades e desafios percebidos pelos discentes das duas primeiras ofertas da disciplina e pelo coletivo docente - professores, gestão acadêmica e assessoria pedagógica - envolvidos no seu planejamento e execução.

\section{Metodologia}

Avaliação qualitativa desenvolvida conforme o referencial teórico-metodológico da Avaliação de Quarta Geração AQG - (Guba \& LINCOLN, 2011), adaptada por Wetzel (2005) e Mielke et al. (2012). Baseada nos pressupostos do paradigma construtivista, sendo a realidade uma construção social, a AQG representa uma avaliação responsiva, onde os marcadores avaliativos são definidos a partir da interação e negociação entre o pesquisador e os grupos de interesse (GI). Participaram deste estudo 35 informantes, dos segmentos docente e discente conforme demonstrado no Quadro 1.

Quadro 1 - Caracterização dos informantes do estudo.

\begin{tabular}{|l|l|l|}
\hline Grupo de Interesse & Caracterização & Quantitativo de informantes \\
\hline Coletivo docente & Professores & 8 \\
\cline { 2 - 3 } & Assessoria Pedagógica & 3 \\
\cline { 2 - 3 } & Gestão Acadêmica & 2 \\
\hline \multirow{4}{*}{ iiscentes } & Ciências Biológicas & 3 \\
\cline { 2 - 3 } & Medicina & 9 \\
\cline { 2 - 3 } & Nutrição & 3 \\
\cline { 2 - 3 } & Terapia Ocupacional & 7 \\
\hline
\end{tabular}

Fonte: Produzido pelos autores do artigo.

O GI discente contemplou os estudantes das duas primeiras turmas, ofertadas no período de julho de 2019 a abril de 2020. A identificação dos GI permitiu que diferentes perspectivas fossem consideradas, de maneira que a inclusão e a concessão de poder no processo investigativo foram elementos característicos na avaliação. 
As informações empíricas foram construídas no período de março/2019 a dezembro;2020. Inicialmente, foi realizada uma etnografia prévia (março/2019 a abril/2020) com a observação das reuniões docentes de planejamento e avaliação das atividades didático-pedagógicas, do desenvolvimento das aulas da disciplina, além da identificação dos GI e seus informantes. As informações obtidas foram registradas no diário de campo da pesquisa. Em seguida, foram realizadas entrevistas semiestruturadas com os informantes da avaliação durante os meses de agosto a novembro/2020. Em decorrência das medidas de distanciamento social causadas pela pandemia de Covid-19, foi impossibilitada a realização de entrevistas presenciais. Como adaptação metodológica, os pesquisadores optaram pela realização de entrevistas por meio do Google Meet. Dada a complexidade e riqueza do material assim obtido, consideramos que essa adaptação metodológica não trouxe prejuízos para a construção dos dados.

A AQG é operacionalizada a partir do Círculo Hermenêutico Dialético (CHD), conforme esquematizado na Figura 1. O CHD iniciou com a entrevista do primeiro informante, ao qual foi solicitado que falasse sobre as potencialidades e desafios da IFISUS. Em seguida, os dados foram analisados visando que o próximo informante, além de apresentar suas perspectivas, discutisse sobre os dados construídos com o informante anterior. Esse processo hermenêutico e dialético foi repetido com todos os informantes do GI. O primeiro CHD foi construído com os discentes, seguido de outro com o coletivo docente. Ao término de cada CHD, os dados foram categorizados em núcleos temáticos e discutidos com os informantes em oficinas de negociação, verificando se as interpretações dos pesquisadores estavam alinhadas com as construções iniciais obtidas, processo que possibilitou reformulações. As oficinas de negociação foram realizadas em outubro e dezembro/2020. Após essa etapa, o material empírico foi organizado em unidades temáticas, permitindo a identificação dos marcadores avaliativos.

Figura 1 - Estrutura esquemática do Círculo Hermenêutico Dialético.

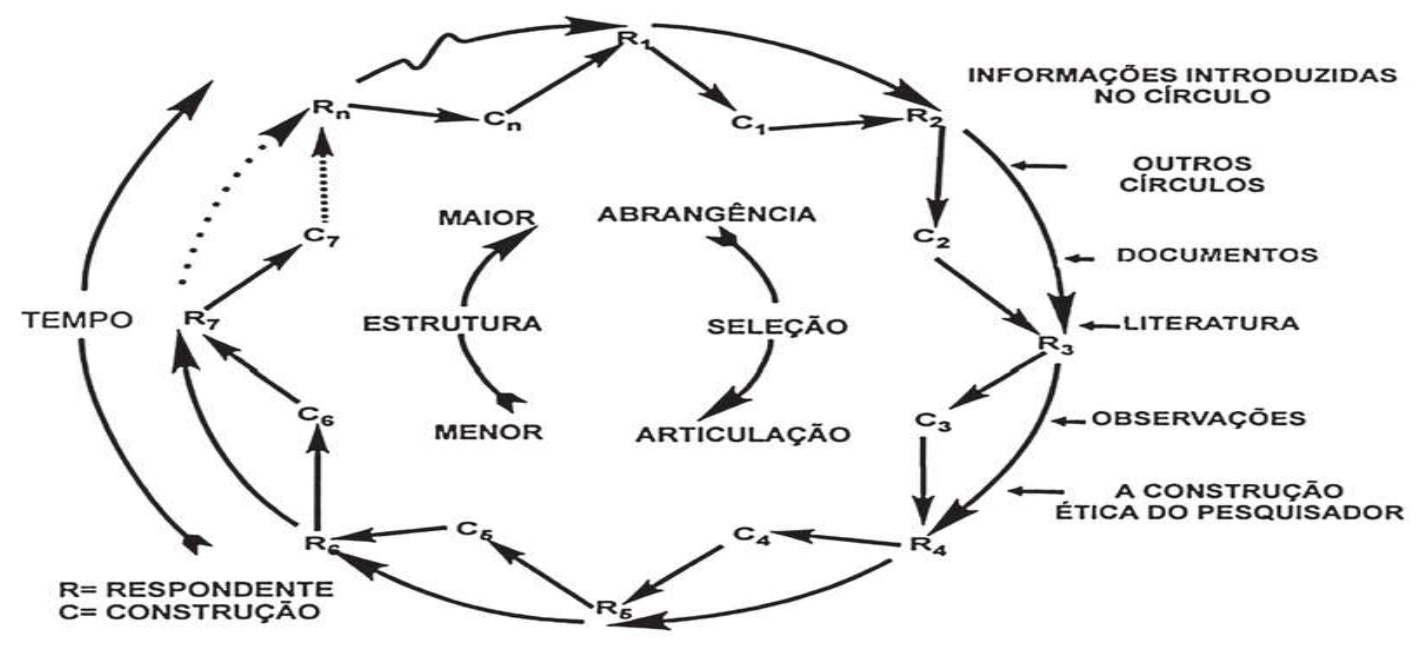

Fonte: Extraído de Guba e Lincoln (2011, p. 169).

A pesquisa cumpriu os princípios e postulados éticos contidos na Resolução no 466 (2012) sendo aprovada pelo Comitê de Ética em Pesquisa da UECE, CAAE: 27238919.0.0000.5534, número do Parecer: 3.799.057.

\section{Resultados e Discussão}

Os resultados contemplam as categorias temáticas potencialidades e desafios da disciplina IFISUS, cujas subcategorias abordamos nos tópicos a seguir. Para facilitar a visualização dos resultados, o Quadro 2 a seguir elenca as categorias e subcategorias que serão apresentadas e discutidas. 
Quadro 2 - Categorias e subcategorias abordadas.

\begin{tabular}{|l|l|}
\hline \multicolumn{1}{|c|}{ Categoria temática } & \multicolumn{1}{c|}{ Subcategorias discutidas } \\
\hline Potencialidades & Oportunidade de aprendizado compartilhado e interativo \\
\cline { 2 - 2 } & Uma formação que valoriza o SUS \\
\cline { 2 - 2 } & Ênfase na dimensão do trabalho em equipe e na colaboração \\
\hline \multirow{2}{*}{ Desafios } & Não adesão de todos os cursos de saúde da IES \\
\cline { 2 - 2 } & Modalidades distintas de oferta curricular da disciplina \\
\cline { 2 - 2 } & A necessidade do desenvolvimento docente para a EIP \\
\hline
\end{tabular}

Fonte: Produzido pelos autores do artigo.

Os depoimentos dos discentes são identificados com a letra "E", seguida do número de ordem da entrevista e as iniciais dos cursos: Ciências Biológicas (B), Medicina (M), Nutrição (N) e Terapia Ocupacional (TO). Da mesma forma, os professores, a assessoria pedagógica e a gestão acadêmica são identificados pelas letras P, AP e GA, respectivamente.

\subsection{Potencialidades}

\subsubsection{Oportunidade de aprendizado compartilhado e interativo}

A IFISUS oportunizou vivências de aprendizado compartilhado e interativo entre discentes de diferentes cursos de saúde, aspecto considerado como um dos pontos mais relevantes da disciplina, enfatizado pelos informantes:

E, essa parte, gostei muito porque aprendi muito com eles, tanto com os colegas de Nutrição, os de Ciências Biológicas, assim como creio que eles aprenderam muito conosco da Medicina [...]. E8M

Toda aula a gente tinha trabalho em grupo, aprender com o outro, conversando, né? Eram diálogos mesmo, debatendo questões do SUS, as dificuldades e tudo. Aí a gente fazia grupos de poucas pessoas e cada um dava sua opinião sobre determinado assunto e entrava em um consenso para explicar para turma depois. E3N

Durante a disciplina a gente conversou bastante, trocamos experiências. Teve até o caso da Terapia Ocupacional, que é um curso novo na UECE. Muita gente não sabia o que eles faziam e a gente conversava, trocávamos experiências. "Onde é que no futuro você pode atuar?" Esse tipo de coisa. E17M

Considerando que essa forma de aprendizado permitiu a troca mútua de conhecimentos e experiências, os discentes apontaram que a vivência com colegas de outros cursos amplia as possibilidades terapêuticas a partir da articulação das diferentes perspectivas de cada profissão, qualificando o cuidado em saúde.

[...] é possível observar diversos pontos de vista. Por exemplo, na definição de um tratamento, é possível identificar diversas oportunidades de tratamento. Então, não somente o tratamento medicamentoso, mas tanto tratamento por meio da alimentação, no caso da nutrição. Esse conjunto de tratamentos que podem se encaixar, promover e causar um benefício muito maior do que apenas um isolado. E1M

Em perspectiva semelhante, os professores apontaram que a IFISUS se constitui como uma estratégia pedagógica que potencializa a formação, ampliando a compreensão dos profissionais de saúde a respeito da vida, mediante o encontro entre 
diferentes profissões:

Um dos aspectos positivos para mim é a possibilidade de gerar encontros entre os diversos campos que compõem o campo maior da saúde [...]. Isso faz com que as formações fiquem um pouco mais potentes, digamos assim. Adquiriram uma complexidade que se aproxima um pouco mais do que a gente chama de vida, a vida cotidiana. Para mim, um ponto positivo da IFISUS é permitir isso, esses encontros, permitir uma formação que considere essas singularidades para que a gente possa ampliar um pouquinho mais o nosso olhar, a nossa percepção. P2

A partir desses relatos acerca do processo de ensino-aprendizagem identificamos a efetivação do compartilhamento de conhecimentos e vivências entre estudantes de diferentes cursos. Nesse sentido, a experiência de aprendizado não se restringiu apenas ao agrupamento de estudantes, mas possibilitou uma formação permeada pela comunicação onde os estudantes aprendem com, para e entre si, articulando diferentes saberes e experiências, ampliando os limites do aprendizado uniprofissional (OMS, 2010). Ademais, esse tipo de aprendizagem tem sido apontada como um espaço profícuo para a formação de profissionais com perfil crítico e reflexivo, proativos e aptos para o trabalho em equipe (Rossit, Freitas, et al., 2018).

Conforme relatado pelo informante E1M, a experiência de EIP possibilitou aos estudantes refletirem sobre o cuidado em saúde a partir de uma perspectiva coletiva. Quando os estudantes aprendem juntos e consideram as diferentes perspectivas de cuidado, vislumbram um horizonte de oportunidades terapêuticas que ultrapassa a perspectiva uniprofissional de produção do cuidado, qualificando a assistência prestada. Relacionamos essa compreensão com os lemas "aprender juntos para trabalhar juntos" e "Aprendendo juntos a trabalhar juntos pela saúde" defendidos nos primórdios da EIP (Costa et al., 2018; OMS, 2010; Peduzzi, 2017).

Iniciativas de aprendizagem compartilhada na perspectiva da EIP devem proporcionar a interação entre os estudantes para o desenvolvimento de competências colaborativas (Costa, 2017). A materialização do aprendizado interativo ocorre quando estudantes participam ativamente, trocando conhecimentos e vivências entre pares (Reeves et al., 2013, 2016). Considerando que a colaboração ocorre quando as diferentes profissões de saúde trabalham de maneira interativa, compartilhando objetivos, reconhecendo a importância do outro e a complementariedade das ações em saúde (Costa, 2017), as falas dos informantes evidenciam que a IFISUS proporcionou um aprendizado pautado pela colaboração.

A fala da informante $\mathrm{E} 3 \mathrm{~N}$ evidencia que os estudantes participaram de momentos formativos que requeriam a abertura para diálogo e a capacidade de escuta como elementos necessários ao aprendizado mútuo. Assim, identificamos uma aproximação com desenvolvimento da competência colaborativa de comunicação interprofissional - competência-chave para a colaboração (Toassi et al., 2020). Isso se associa com a compreensão que a EIP proporciona aos estudantes de graduação o desenvolvimento da comunicação interprofissional, o que qualifica as decisões clínicas das equipes de saúde (Stokes et al., 2020).

Além dessa competência colaborativa, identificamos nos relatos uma aproximação com a competência da clarificação dos papéis. Como trazido pela informante E17M, a experiência na IFISUS contribuiu para que os estudantes aprendessem algo, compreendessem mais sobre a área e atuação das demais categorias. Corroborando esse achado, estudos evidenciam que a EIP contribuiu para que os estudantes compreendessem melhor sobre o trabalho das diferentes profissões (Toassi et al., 2020), promovendo a desconstrução de estereótipos profissionais negativos que são barreiras para o trabalho em equipe. Quando profissionais reconhecem os papéis e as responsabilidades das demais profissões passam a ter uma visão mais abrangente e clara sobre como cada profissional pode contribuir para o cuidado prestado (Peduzzi, 2017). 


\subsubsection{Uma formação que valoriza o SUS}

Outro aspecto positivo da IFISUS relatado pelos informantes é o desenvolvimento de um processo formativo que valoriza o SUS. Na perspectiva do coletivo docente, a opção de trabalhar os marcos teórico-conceituais do SUS, representa a intencionalidade pedagógica da disciplina em contextualizar a formação dos profissionais de saúde com essa importante política pública, trabalhando conceitos como a compreensão ampliada do processo saúde-doença-cuidado, os princípios e diretrizes do SUS e a relação desses conceitos com a atuação profissional nos serviços de saúde.

Primeira coisa, [é] como ela foi pensada. [Foi] a partir da proposta da própria criação do SUS, do desenho do SUS, onde os cursos da área da saúde precisavam formar profissionais para atuar no SUS. GA1

Da experiência com o SUS como um todo, desde o seu arcabouço teórico, e entra nesse arcabouço teórico [que] ele não é só no sentido de dizer o que é o SUS e suas diretrizes, mas, inclusive, as próprias concepções relacionadas ao processo saúde-doença que sustentam o SUS, que embasa o conceito de saúde que está presente lá e tem em alguns documentos, inclusive, na própria Lei Orgânica da Saúde. [...] não só no ponto de vista teórico, mas prático também, no sentido de sempre pensar como é que tudo isso se materializa no cotidiano de trabalhadores do SUS. P6

Efeitos dessa intencionalidade pedagógica foram percebidos nas falas dos estudantes, para quem um dos aspectos positivos da disciplina é contemplar na formação conhecimentos relacionados ao SUS, desde o processo histórico que culminou em sua criação, assim como os princípios e diretrizes que norteiam e operacionalizam as ações de saúde desenvolvidas em seu âmbito.

Outro ponto muito interessante foi toda a história, a apresentação do SUS para a gente, porque muitas pessoas não têm conhecimento da importância e grandiosidade do SUS. E13TO

Achei uma abordagem muito diferente pois desde o primeiro momento a gente já tem um contato com o SUS, o que ele é, os meus direitos e deveres como usuário. Então acho que é um momento muito importante dentro da instituição, porque a gente entra sem ter muito contato com o que de fato é o mecanismo de funcionamento do SUS E16M

A constatação que "desde o primeiro momento a gente já tem um contato com o SUS", é amparada na disciplina de maneira teórica e prática; a IFISUS contempla vivências de EIP nos serviços da APS de Fortaleza, aproximando os estudantes com a realidade e a dinâmica de funcionamento desses serviços, a complexidade do trabalho dos profissionais, seus territórios de atuação, usuários atendidos e suas demandas de saúde. Ao diversificar o cenário de ensino-aprendizagem, transpondo os limites da sala de aula, alcançando o local de materialização das práticas profissionais e os contextos de vida daqueles que utilizam o SUS, percebemos na IFISUS uma aproximação entre ensino, serviço e comunidade.

Essa contextualização da formação em saúde com o SUS colaborou para que os estudantes assumissem uma postura favorável à sua defesa e valorização, como explicitado nas seguintes falas:

[...] a disciplina foi importante para que tivéssemos argumentos do porquê que defendemos o SUS, por que é um sistema tão importante e por que precisa ser preservado. E15TO

Para mim, [o ponto mais importante] foi a maior valorização do SUS. Cheguei na disciplina com uma ideia totalmente 
diferente sobre o que era o SUS. Eu julgava uma coisa que não conhecia, a história de como era antes dele, depois de sua criação e como era em outros países. Então, saí da disciplina com uma maior valorização do SUS. Hoje eu defendo esse sistema. E21M

A reflexão sobre as falas dos estudantes e professores nos permite compreender que a disciplina proporcionou situações de ensino-aprendizagem centrados na formação profissional para e a partir do SUS. Nos relatos discentes percebemos que a abordagem das temáticas relacionadas ao SUS não se restringiu à uma concepção conteudista de exposição às informações legais e normativas. Em uma reflexão mais profunda, é possível perceber elementos relacionados com o desenvolvimento de uma aprendizagem significativa, tais como a mobilização de dimensões cognitivas, subjetivas e emocionais, articulando a teoria e prática de maneira crítica e reflexiva (Azevedo et al., 2019; Lemos, 2019).

É a partir dessas reflexões que associamos a IFISUS aos movimentos de reorientação da formação em saúde no Brasil, tais como as DCN, o Pró-Saúde e o Programa de Educação para o Trabalho em Saúde (PET-Saúde), que enfatizam a necessidade das IES pautarem a formação profissional a partir de uma abordagem integral do processo saúde-doença-cuidado, com ênfase nos princípios e diretrizes do SUS, na valorização da APS e na integração ensino-serviço-comunidade, como elementos essenciais para o fortalecimento do SUS (Brasil, 2018a; 2001; Moraes, 2016).

Conforme Campos (2018) e Paim (2018), apesar de sua implantação e regulamentação, o SUS ainda se depara com os desafios de consolidação e sustentabilidade. Conforme os autores, o enfrentamento desses desafios requer a formação de profissionais com competências alinhadas com os seus princípios e diretrizes estabelecidas. Nesse caso, identificamos que a IFISUS contribuiu com a formação em saúde ao proporcionar que aos estudantes o desenvolvimento de conhecimentos necessários à prática profissional para e a partir do SUS, assim como a atitude de valorização e defesa dessa política de saúde.

\subsection{3 Ênfase na dimensão do trabalho em equipe e na colaboração}

Outra potencialidade da IFISUS está relacionada à valorização do trabalho em equipe. Na perspectiva dos estudantes, a vivência na disciplina proporcionou a compreensão que o trabalho em saúde não se reduz à atuação específica de um profissional, mas pelo trabalho coletivo de diferentes categorias. Além disso, eles compreenderam que a oferta de cuidado integral demanda uma lógica de atuação balizada pela interprofissionalidade. Os depoimentos a seguir evidenciam essa compreensão:

A questão do trabalho em equipe é, de fato, um dos pontos mais importantes do profissional de saúde, porque tem que sempre ocorrer aquela interação de várias profissões para o atendimento da população, atendimento do paciente. E1M

Pude perceber na disciplina que a interprofissionalidade é, de fato, o coração do SUS. A integralidade só vai ser garantida se a gente tiver essa interprofissionalidade. [...] Por exemplo, tem um paciente com dificuldade de locomoção. Eu como médica vou poder receitar um tratamento para ele, mas eu sei que o melhor tratamento para ele vai ser o que um fisioterapeuta vai poder dar. Então, para garantir a integralidade, garantir que o serviço seja completo, e o mais adequado possível para o paciente, eu vejo o quanto essa interprofissionalidade vai ser importante. E7M

Essa compreensão sobre a importância do trabalho em equipe foi associada com o reconhecimento de uma interdependência entre os profissionais de saúde, de maneira tal que a atuação de um dado profissional necessita e é complementada pelo trabalho do outro. 
A questão não é o grau de importância de cada profissão. A gente entende, assim como no corpo, eu não posso dizer que a minha perna é menos importante do que os meus olhos. Cada um tem a sua função. Sem as minhas pernas eu não consigo me locomover. Sem os meus olhos eu não consigo ver. E11M

Não acredito na ideia de que a gente consegue [atuar na saúde] sozinho. Acredito que para a gente conseguir uma melhora, um bom tratamento, uma recuperação, atingir o nosso objetivo real, a gente precisa trabalhar em conjunto. E14TO

De maneira semelhante, a temática do trabalho em equipe e a dependência mútua entre as profissões de saúde foi um tema recorrente nas falas dos informantes da assessoria pedagógica:

[Um dos aspectos positivos dessa disciplina] é a gente introduzir no primeiro semestre de um curso de saúde essa visão do SUS e da saúde ampliada, o trabalho em equipe, a colaboração. [...] é criar neles esse espírito colaborativo que hoje, para mim, vai além do SUS. [...] Eu sou restrito, por natureza humana, e preciso do outro para me completar. Então, isso, para mim, traz um benefício humano, [uma] formação humana muito grande. AP1

Conforme os relatos, o aprendizado interprofissional contribuiu para os participantes perceberem o trabalho em equipe como uma modalidade que resulta em maiores benefícios aos usuários dos serviços de saúde quando comparada à atuação uniprofissional. Isso revela a positividade da EIP para a formação de profissionais mais aptos ao desenvolvimento do efetivo trabalho em equipe no SUS. Em geral, estudantes que vivenciaram experiências de EIP consideram que o aprendizado com colegas de outros cursos os torna mais habilitados para o trabalho em equipe (Aguilar-da-Silva et al., 2011).

Corroborando esses achados, estudos sustentam que as oportunidades de EIP na graduação proporcionam o desenvolvimento de competências necessárias para o efetivo trabalho em equipe e a adoção de práticas integrais de cuidado, resultando na melhoria da qualidade da assistência ofertada (Freire Filho et al., 2019; Moraes et al., 2019; Toassi et al., 2020). Segundo Costa (2017), o trabalho em equipe é um imperativo para o enfrentamento das complexas e dinâmicas demandas de saúde.

Nessa compreensão, processos formativos que enfatizam o trabalho em equipe e o cuidado integral, à semelhança da IFISUS, contribuem para a construção de um perfil profissional não restrito ao desenvolvimento de conhecimentos e práticas profissionais específicas, mas que avançam na formação de um profissional mais colaborativo (Rossit, Freitas, et al., 2018).

Conforme Costa (2017) e D’Amour et al. (2005), dentre os elementos necessários à colaboração entre profissionais estão o compartilhamento, a parceria e a interdependência. Com base nos relatos dos informantes, observamos que a IFISUS proporcionou aos estudantes aproximação com esses elementos. O compartilhamento foi trabalhado na medida em que os estudantes compartilhavam conhecimentos e vivências prévias, bases teóricas e conceituais na graduação. A parceria, por sua vez, embora os autores supracitados a relacionem mais com a prática profissional, foi também percebida nos relatos onde os estudantes reconhecem e valorizam a importância das demais profissões de saúde. Por último, a interdependência foi evidenciada na medida em que os estudantes reconhecem os limites da atuação profissional isolada, assumindo a necessidade da complementaridade dos saberes e práticas no cuidado em saúde.

Quando estudantes e profissionais de saúde exercitam a interdependência e complementaridade, parceria, comunicação efetiva e respeito mútuo entre as profissões, se distanciam de modelos tradicionais de ensino e trabalho caracterizados pela fragmentação dos saberes e das práticas em saúde, percorrendo caminhos de formação e prática 
profissional mais colaborativas (Costa, Freire Filho, et al., 2018; Rossit, Freitas, et al., 2018). Tais reflexões nos permitem considerar que a EIP é uma possibilidade real para a formação de profissionais de saúde mais aptos ao exercício da colaboração e do trabalho em equipe.

\subsection{Desafios}

\subsubsection{Não adesão de todos os cursos de saúde da IES}

Um dos desafios da disciplina IFISUS apontado pelos informantes está relacionado com a não adesão de alguns cursos de saúde da IES à proposta de EIP:

Uma falta que eu senti na disciplina foi a ausência do pessoal do curso de enfermagem, que também está dentro do CCS [...]. E1M

Não é que não tenha ficado claro. A gente sempre lia e via que a interprofissionalidade é essencial. Só que eu acho que o que realmente faltou foi trazer outros cursos. Por exemplo, se trouxesse a enfermagem, já daria algo a mais. E7M

De maneira semelhante aos relatos discentes, os docentes consideram que a não adesão de outros cursos de saúde na IFISUS, não somente os do CCS, mas também de outros centros, é um desafio para o desenvolvimento da EIP.

Não ter todos os cursos da saúde [participando da disciplina], para mim, é um ponto negativo. Deveria já ter envolvido todos os cursos da área da saúde que compõem a UECE. P2

Por exemplo, a gente se restringiu muito, na minha experiência, ao CCS. E a gente tem muito a aprender com quem vem lá do Centro de Estudos Sociais Aplicados, com o Serviço Social, que é uma profissão que está hoje muito presente no SUS. [O curso de] Psicologia também. E esses profissionais às vezes, até saem sem saber, conhecer o SUS. P6

Ao serem indagados sobre os motivos da não adesão dos demais cursos à disciplina, informantes do coletivo docente relacionaram diferentes razões, entre estas, a falta de apoio institucional, resistência das coordenações dos cursos e Núcleos Docentes Estruturante (NDE), além do desinteresse de alguns cursos com a EIP:

Falta um processo de gestão central que seja capaz de definir "todos os cursos vão participar" ou "tais e tais cursos vão participar”. P7

[...] pouco apreço que algumas coordenações de cursos têm em relação à IFISUS. P2

E os próprios alunos percebem isso: "por que que a enfermagem não está aqui? Por que que a psicologia não está aqui?”. [...] E a gente tem que dizer que não estão porque não querem. Não estão porque não se organizaram para viabilizar aquilo. E a gente sabe que não se organizaram porque não é uma prioridade do curso ter essa disciplina. P7

Outra coisa negativa é a disciplina não ser assumida pelos NDE de todos os cursos. É um grupo que se sensibiliza. 
Embora, a gênese dessa disciplina seja em oficinas do NDE. Mas, o NDE assumiu [à época], disse que ia ser obrigatória, mas quando chega nos conselhos dos cursos, isso não é efetivado. AP1

Contudo, em perspectiva divergente, uma informante da gestão acadêmica sustentou que a dificuldade de inserir a disciplina IFISUS nos currículos de alguns cursos está relacionada aos momentos distintos dos diferentes cursos quanto ao processo de reestruturação de seus projetos pedagógicos (PPC).

Nós ainda temos cursos que estão ausentes, e nós gostaríamos que todos estivessem lá presentes. Então, esse processo de reestruturação dos projetos pedagógicos, eles não caminham no mesmo passo. Isso porque essa reestruturação dos projetos pedagógicos como uma readequação para a formação dos profissionais de saúde não foi incorporada ainda pela Pró-Reitoria de Graduação. Ela ainda não incorporou isso como uma meta pedagógica da universidade. Nós ainda sentimos muito isso. Isso foi tomado com uma bandeira da área da saúde, mas não incorporada pela instituição. Então, isso dificulta com que isso aconteça. GA1

Essas divergências revelam que a inserção da EIP nos currículos em saúde se constitui como um processo complexo e multifacetado, e não implementado sem a presença de resistências. Assumir a EIP como uma diretriz para a reorientação da formação em saúde e o ordenamento da formação de profissionais colaborativos e mais aptos para elevar a qualidade da atenção prestada, é também tensionar um modelo formativo tradicional, fragmentado, com ênfase nas competências específicas, as quais estimulam a atuação profissional individualista, a formação de estereótipos profissionais e desconhecimento dos papéis e responsabilidades das demais profissões (Almeida et al., 2019; Ely \& Toassi, 2018; Toassi et al., 2020). Esse modelo formativo tem sido legitimado e perpetuado por meio de desenhos curriculares conservadores centrados em abordagens ainda disciplinares, com pouca ênfase no desenvolvimento de competências comuns e ausência de componentes curriculares que proporcionem o desenvolvimento das competências colaborativas. Tais currículos dificultam a adoção e desenvolvimento de projetos de EIP (Brasil, 2018b).

Nesse contexto se percebe a importância do apoio institucional para a implantação e desenvolvimentos de iniciativas pedagógicas pautadas pela interprofissionalidade. Tendo em vista que a estrutura departamental das IES e os currículos uniprofissionais inflexíveis são barreiras para as iniciativas de EIP, a adoção de políticas institucionais e comprometimento administrativo em apoio a esse tipo de aprendizado são elementos cruciais para o sucesso dessas iniciativas (Reeves, 2016). No caso da IES estudada, a estrutura não é departamental, mas por colegiados reunidos em torno de centros de estudos e existem cursos que formam categorias profissionais em diferentes centros. A proposta de EIP como uma iniciativa do CCS foi aceita, mas não adotada como uma proposta pedagógica da IES para a formação em saúde, concorrendo para a adesão à mesma ser uma opção dos cursos.

A ausência dos cursos de Educação Física, Enfermagem, Psicologia, Medicina Veterinária e Serviço Social existentes na IES revela aspectos contextuais que limitam a inserção da EIP na formação, tais como a rigidez dos currículos uniprofissionais, a morosidade na reestruturação e implantação dos PPC e insuficiente apoio institucional que garanta a integração de outros cursos e agilize os trâmites burocráticos. Esses aspectos evidenciam um cenário institucional com resistências à mudança, ao novo, e, consequentemente, à efetivação da EIP como diretriz para a formação em saúde.

Entretanto, é importante considerar que a integração de outros cursos à experiência de EIP traz consigo o complexo desafio de delimitar a(s) interface(s) entre as diferentes áreas profissionais para o desenvolvimento da prática interprofissional colaborativa (PIC), onde as diferentes profissões compartilham conhecimentos, habilidades e expertises para prover um cuidado centrado nos usuários (Batista \& Peduzzi, 2018). Sobre as PIC, não identificamos situações nas quais os estudantes da 
IFISUS tenham desenvolvido tais práticas junto aos usuários dos serviços de saúde, o que representa uma importante lacuna nessa recente disciplina de EIP, mas também aponta para a necessidade de fortalecimento da articulação ensino-serviço no sentido de integrar o serviço como parceiro na formação dos profissionais de saúde.

Ely e Toassi (2018) referem que a qualificação da EIP demanda um apoio institucional consistente por meio de políticas e recursos para o desenvolvimento dessas inciativas, sensibilização e articulação dos sujeitos e dispositivos institucionais envolvidos com a formação em saúde, disseminação dos conhecimentos sobre a interprofissionalidade e dos resultados das experiências de EIP.

No cenário estudado, a iniciativa de implantar a EIP partiu do CCS por uma visão da gestão do centro quanto à sua potencialidade enquanto proposta de reorientação do modelo formador. Contudo a ausência do amplo apoio institucional, foi determinante para os desafios identificados:

[É um aspecto positivo] o apoio da direção do CCS, porque sem ele seria realmente muito difícil conseguir isso. Porque teve que interferir nos projetos pedagógicos dos cursos, que é uma resistência muito grande. P8

Mediante os achados promissores deste estudo, evidencia-se a necessidade da adoção de estratégias para sensibilização e envolvimento dos demais centros e coordenações de cursos para a adoção da EIP, bem como a tornar uma pauta prioritária junto à administração superior da IES, visando a adoção de mecanismos institucionais facilitadores da adesão dos demais cursos de saúde, alternativa viável, como demonstrado pela experiência da IFISUS de superação do modelo formador fragmentado, o que, é claro, será um processo complexo e desafiador, mas necessário.

\subsubsection{Modalidades distintas de oferta curricular da disciplina}

Um outro desafio identificado pelos informantes é a diversidade na modalidade de oferta da IFISUS, podendo ser como uma disciplina obrigatória, optativa ou atividade complementar, o que também guarda relação com os momentos distintos vivenciados pelos cursos no processo de reestruturação dos PPC. Para a assessoria pedagógica e professores, essa diversidade poderia, inclusive, interferir no rendimento dos estudantes na disciplina:

Para mim, uma dificuldade e algo que é ruim, é essa falta de assumir que a disciplina é obrigatória para todos. Então, a gente teve que viver essa realidade. É uma dificuldade você ter em uma sala alunos que são matriculados em uma disciplina complementar, que é optativa, e [para] os outros que é obrigatória. Humanamente falando - e não é uma questão do aluno, é uma questão humana -, você percebe que é dificultoso para o professor e para os alunos, para a convivência. E se a gente vai trabalhar com equipe, com colaboração, isso traz uma dificuldade muito grande para a gente trabalhar. AP1

Mas, com certeza, porque a gente também observava essa questão de diferença dos alunos quando é uma disciplina obrigatória ou quando conta só como uma carga horária complementar. Tinha essa diferença na participação durante a disciplina. P3

Incialmente, o grupo docente engajado na construção da IFISUS desejava que a disciplina fosse inserida nos currículos dos cursos de saúde como obrigatória. Entretanto, no decorrer do processo de sua inserção nos currículos, ela foi inserida como disciplina obrigatória apenas nos cursos de Medicina e Ciências Biológicas. Na matriz curricular do curso de Terapia Ocupacional, recém implantado na IES, cuja primeira turma coincidiu com a primeira oferta da disciplina, esta entrou 
como optativa, forma da coordenação do curso viabilizar sua oferta; já ocurso de Nutrição a viabilizou como atividade complementar. O curso passava então pelo processo de reestruturação curricular. Assim, como estratégia para não limitar ainda mais os cursos envolvidos com a experiência de EIP, a coordenação do curso sugeriu que a IFISUS fosse ofertada como atividade complementar até a adoção da nova matriz curricular.

A respeito disso, é reconhecido que a oferta obrigatória da EIP é um mecanismo curricular que aperfeiçoa o desenvolvimento desse tipo de aprendizagem e da prática colaborativa (OMS, 2010). Estudantes que a vivenciaram consideram que essas vivências deveriam ser ofertadas na modalidade obrigatória nos currículos de saúde (Ely \& Toassi, 2018; Toassi et al., 2020).

As diferenças entre as modalidades de oferta, como existentes na IFISUS, podem fazer com que existam questionamentos a respeito do valor dessas inciativas para a formação profissional (Ely \& Toassi, 2018; Reeves, 2016; Toassi et al., 2020). Assim, ao considerarmos os relatos sobre as diferenças no rendimento dos estudantes na IFISUS e as recomendações para a oferta da EIP, consideramos que a oferta obrigatória dessa disciplina para os cursos da saúde representaria uma estratégia que qualificaria esse tipo de aprendizado. Contudo, os resultados positivos abordados anteriormente apontam que a participação dos cursos, os quais, circunstancialmente não conseguiram iniciar a disciplina como obrigatória, favoreceu o aprendizado interprofissional vivenciado e demonstrou a importância da sua inserção na matriz curricular.

\subsubsection{A necessidade do desenvolvimento docente para a EIP}

Outro aspecto desafiador expresso por alguns informantes é sobre a necessidade de estratégias de desenvolvimento docente para o exercício da EIP:

Isso é uma grande fragilidade. [...] Sem fazer o desenvolvimento docente para a Educação Interprofissional e para o trabalho profissional no serviço, nós na verdade não vamos avançar. Esse desenvolvimento docente é fundamental. GA2

A outra questão é a nossa própria formação como docente. Eu sinto falta de ser formada. Assim, como se os nossos encontros fossem não só para planejar aula, mas para a gente também aprender a conduzir interprofissionalmente, porque eu estudei sobre interprofissionalidade no mestrado, fiz residência. [...] Mas, eu sinto necessidade de, além do conteúdo da disciplina, ter que estudar e ser formada mesmo sobre como trabalhar, como ministrar uma aula focando na interprofissionalidade, sabe? Como trabalhar com grupos, essa coisa que não é tão fácil, né? P7.

O coletivo docente da IFISUS é formado por professores com formações disciplinares e que lecionam nos diversos cursos de saúde da IES, o que se demonstrou como uma característica potente para a condução da EIP, mas a atividade docente nessa perspectiva de ensino-aprendizagem demanda um processo contínuo e crítico de desenvolvimento docente (Santos et al., 2018). Batista et al. (2018) apontam que um dos problemas existentes nas IES é a ênfase nas métricas individuais de pesquisa em detrimento do ensino. Além disso, é comum que os professores não tenham recebido uma formação que os qualifiquem para o desenvolvimento da EIP (Brasil, 2018b).

Diante desse cenário, constitui-se como um imperativo a intensificação de esforços institucionais para a qualificação docente, valorizando o ensino na graduação e um maior envolvimento dos professores com os PPC, aprimorando os currículos para a adoção de estratégias pedagógicas inovadoras, valorizando a articulação dos diferentes saberes, e a integração entre as IES e os dispositivos de saúde do SUS (Brasil, 2018b). 
O desenvolvimento do corpo docente é decisivo para o sucesso da EIP (Barr, 2015; El-Awaisi et al., 2016; Reeves, 2016). É um desafio comum para os docentes o ensino na perspectiva da EIP, uma vez que foram formados em uma lógica uniprofissional. Assim, a superação desse desafio demanda a ampliação da competência docente para facilitar/mediar oportunidades de EIP. Essa competência abrange as experiências prévias com a interprofissionalidade, a intencionalidade para o trabalho em grupo interprofissional colaborativo, conhecimento profundo em estratégias interativas de aprendizado, flexibilidade e criatividade para as vivências de aprendizado compartilhado, envolvimento e compromisso com a EIP (Poletto \& Jurdi, 2018; Reeves, 2016; Santos et al., 2018).

No contexto estudado, identificamos a ausência de um programa institucional de alinhamento docente com os marcos teóricos, conceituais e metodológicos da EIP, o que em parte, tem sido preenchida a partir do próprio processo de planejamento, implantação e desenvolvimento da IFISUS, tal como expresso a seguir:

Nós não temos uma formação docente prévia para ensinar essa disciplina inovadora. Então, na minha forma de enxergar, os professores estão em um processo formativo. AP1

As observações em sala de aula e nas reuniões com o grupo de docentes reafirmam esta fala. Dentre as contribuições da disciplina para a qualificação da atividade docente, entre outras, ressaltamos: a experiência da construção colaborativa de uma disciplina de EIP; o compartilhamento mútuo de conhecimentos e experiências prévias de cada docente com a interprofissionalidade, tanto na dimensão do trabalho quanto da formação; a parceria docente interprofissional, onde uma dupla ou trio de professores articula entre si seus diferentes saberes para facilitação da aprendizagem em cada turma da disciplina; as contribuições advindas da assessoria pedagógica, trazendo elementos conceituais e metodológicos do campo da pedagogia e, particularmente, da didática, para a adoção de estratégias de ensino-aprendizagem alinhadas com a EIP.

Contudo, apesar da potência advinda dessa vivência interprofissional para a prática docente, não se extingue a demanda de uma política institucional de qualificação docente alinhada com essa forma de desenvolver o processo ensino/ aprendizagem. Sustentamos essa perspectiva ao defendermos que a implementação da IFISUS tem se revelado como um processo que, embora fecundo, é complexo, desafiador e marcado por contradições, tais como: a dificuldade de articular outros cursos de saúde da UECE para inserir a EIP nos PPC; as distintas ofertas da disciplina (obrigatória, optativa e atividade complementar); ausência de continuidade da EIP no contínuo formativo do estudante; necessidade de maior apoio e articulação institucional para o seu desenvolvimento.

Como benefícios advindos da adoção de estratégias de desenvolvimento do corpo docente para a promoção da EIP, citamos: integração de professores de distintas áreas profissionais e cursos da saúde para pensar e discutir propostas de aprendizado colaborativas e interativas; criação de espaços institucionais para o diálogo e aprendizado entre docentes; a reflexão sobre os papéis e responsabilidades das categorias de saúde; perceber um campo comum para o ensino da saúde; promover no contexto da IES uma cultura de trabalho em equipe entre os docentes de diferentes cursos (Costa, Freire Filho, et al., 2018; Costa, Peduzzi, et al., 2018).

Desse modo, é crucial que as IES proporcionem espaços de desenvolvimento docente para a discussão sobre os marcos teóricos, conceituais e metodológicos da EIP e da prática colaborativa centrada nos usuários, as teorias educacionais pertinentes e as estratégias de ensino-aprendizagem que facilitam principalmente o desenvolvimento das competências colaborativas, assim como as competências comuns e específicas (Brasil, 2018b; Costa, 2017; Reeves et al., 2016).

\section{Considerações Finais}

Os resultados apresentados nesse estudo nos permitem identificar um alinhamento da disciplina IFISUS com os fundamentos teóricos, conceituais e metodológicos da EIP, uma vez que ela oportunizou experiências de aprendizado 
compartilhado e interativo entre estudantes de diferentes cursos da saúde, com a efetivação de um percurso formativo centrado no SUS, no trabalho em equipe e na colaboração entre os profissionais de saúde para a oferta de um cuidado integral. Todavia, a efetivação da EIP não ocorre sem desafios institucionais que limitam o seu alcance e efeitos na formação em saúde, o que evidencia a necessidade da implantação da EIP integrar a agenda institucional. É importante que a sua oferta ocorra transversalmente nos currículos dos cursos de saúde da UECE, não apenas de maneira pontual, expandindo esse tipo de aprendizado para disciplinas comuns entre os cursos e em estágios curriculares.

O desenvolvimento desse estudo é oportuno quando consideramos o interesse e o investimento crescente em iniciativas de EIP por professores, profissionais de saúde, pesquisadores e formuladores de políticas públicas no Brasil e ao redor do mundo. Esse estudo contribui com a base de evidências sobre experiências de EIP, um tema que requer mais pesquisas sobre sua adoção pelas IES, como estratégia potente de mudança do modelo formador na área da saúde. São necessários estudos que avaliem como a EIP pode contribuir para a adoção das PIC junto aos usuários, expandindo as investigações com cursos e IES brasileiras, buscando aprofundar o conhecimento sobre a EIP e sua influência nas transformações das práticas profissionais nos serviços de saúde.

Uma limitação neste estudo, dado o referencial metodológico, foi a não inclusão do serviço, profissionais e gestores, como grupo de interesse, o que foi circunstancial, dada a avaliação ser do processo de implantação da EIP na IES, não havendo ainda possíveis reflexos para a prática nos serviços. Fica, portanto, a pista para novos estudos.

\section{Referências}

Aguilar-da-Silva, R. H, Scapin, L. T., \& Batista, N. Alves. (2011). Avaliação da formação interprofissional no ensino superior em saúde: Aspectos da colaboração e do trabalho em equipe. Avaliação: Revista da Avaliação da Educação Superior (Campinas), 16 (1), 165-184. https://doi.org/10.1590/S141440772011000100009 .

Almeida, R. G dos S., Teston, E. F., \& Medeiros, A. de A. (2019). Uma interface entre o pet-saúde / interprofissionalidade e a política nacional de educação permanente em saúde. Saúde em Debate, 43 (esp. 1), 97-105. https://doi.org/10.1590/0103-11042019s108.

Azevedo, S. B, Pacheco, V. A., \& Santos, E. A dos. (2019). Metodologias ativas no ensino superior: Percepção de docentes em uma instituição privada do Distrito Federal. Revista Docência do Ensino Superior, 9, 1-22. https://doi.org/10.35699/2237-5864.2019.2573

Barr, H. (2015) Educação interprofissional - a gênese do movimento global. (2016). CAIPE. https://www.caipe.org/resources/publications/barr-h-2015interprofessional-education-genesis-global-movement.

Batista, N. A., Rossit, R. A. S., Batista, S. H. S. da S., Silva, C. C. B. da, Uchôa-Figueiredo, L. da R., \& Poletto, P. R. (2018). Educação interprofissional na formação em Saúde: A experiência da Universidade Federal de São Paulo, campus Baixada Santista, Santos, Brasil. Interface - Comunicação, Saúde, Educação, 22 (supl 2), 1705-1715. https://doi.org/10.1590/1807-57622017.0693.

Batista, R. E. A., \& Peduzzi, M. (2018). Prática interprofissional colaborativa no serviço de emergência: Atribuições privativas e compartilhadas dos fisioterapeutas. Interface - Comunicação, Saúde, Educação, 22(2), 1685-1695. https://doi.org/10.1590/1807-57622017.0755.

Brasil (1990). Lei no 8.080, de 19 de setembro de 1990. Dispõe sobre as condições para promoção, proteção e recuperação da saúde, a organização e o funcionamento dos serviços correspondentes e dá outras providências, 19 (1990). https://portalarquivos2.saude.gov.br/images/pdf/2015/setembro/30/Lei8080.pdf.

Brasil (2001). Parecer no 1.133 de 7 de outubro de 2001. Dispõe as Diretrizes Curriculares para os cursos de graduação de Enfermagem, Medicina e Nutrição, Parecer no 1.133 de 7 de outubro de 2001. Dispõe as Diretrizes Curriculares para os cursos de graduação de Enfermagem, Medicina e Nutrição 39 (2001). http://portal.mec.gov.br/cne/arquivos/pdf/2001/pces1133_01.pdf.

Brasil (2012). Resolução no 466, de 12 de dezembro de 2012. Aprova as diretrizes e normas regulamentadoras de pesquisas envolvendo seres humanos, 12 (2012). https://conselho.saude.gov.br/resolucoes/2012/Reso466.pdf.

Brasil (2018a). Construindo caminhos possíveis para a Educação Interprofissional em Saúde nas Instituições de Ensino Superior do Brasil. Brasília: Ministério da Saúde. https://www.educacioninterprofesional.org/sites/default/files/fulltext/2018/2018_DEGES_construindo_caminhos_EIP.pdf.

Brasil (2018b). Laboratório de Inovação em Educação na Saúde com ênfase em Educação Permanente. Brasília: Ministério da Saúde. https://apsredes.org/wpcontent/uploads/2018/07/NavegadorSUS-WEB-INTER.pdf.

Campos, G. W. de S. (2018). SUS: O que e como fazer? Ciência \& Saúde Coletiva, 23 (6), 1707-1714. https://doi.org/10.1590/1413-81232018236.05582018.

Capozollo, A. A. (2017). Formação em comum de profissionais da saúde: experiência da UNIFESP, campus baixada santista. In R. F. C. Toassi (Ed.), Interprofissionalidade e formação na saúde: onde estamos? (pp. 68-80). Porto Alegre: Rede Unida. http://historico.redeunida.org.br/editora/bibliotecadigital/serie-vivencias-em-educacao-na-saude/vol-06-interprofissionalidade-e-formacao-na-saude-pdf. 
Ceccim, R. B. (2017). Interprofissionalidade e experiências de aprendizagem: inovações no cenário brasileiro. In R. F. C. Toassi (Ed.), Interprofissionalidade e formação na saúde: onde estamos? (pp. 49-67). Porto Alegre: Rede Unida. http://historico.redeunida.org.br/editora/biblioteca-digital/serie-vivencias-emeducacao-na-saude/vol-06-interprofissionalidade-e-formacao-na-saude-pdf.

Ceccim, R. B. (2018). Conexões e fronteiras da interprofissionalidade: Forma e formação. Interface - Comunicação, Saúde, Educação, 22 (2), 17391749. https://doi.org/10.1590/1807-57622018.0477.

Costa, M. V. da. (2017). A potência da educação interprofissional para o desenvolvimento de competências colaborativas no trabalho em saúde. In R. F. C. Toassi (Ed.), Interprofissionalidade e formação na saúde: onde estamos? (pp. 49-67). Porto Alegre: Rede Unida. http://historico.redeunida.org.br/editora/biblioteca-digital/serie-vivencias-em-educacao-na-saude/vol-06-interprofissionalidade-e-formacao-na-saude-pdf.

Costa, M. V. da, Freire Filho, J. R., Brandão, C., \& Silva, J. A. M. da. (2018). A Educação e o trabalho interprofissional comprometimento histórico de fortalecimento e consolidação do Sistema Único de Saúde (Sus). Interface - Comunicação, Saúde, Educação, 22 (2), 1507-1510. https://doi.org/10.1590/180757622018.0636

Costa, M. V. da, Peduzzi, M., Freire Filho, J. R., \& Silva, C. B. G. (2018). Educação Interprofissional em Saúde. Natal: SEDIS-UFRN. http://portalarquivos2.saude.gov.br/images/pdf/2018/dezembro/12/Educacao-Interprofissional-em-Saude.pdf.

D'Amour, D., Ferrada-Videla, M., San Martin Rodriguez, L., \& Beaulieu, M.-D. (2005). A base conceitual para a colaboração interprofissional: Conceitos centrais e referenciais teóricos. Journal of Interprofessional Care, 19 (sup1), 116-131. https://doi.org/10.1080/13561820500082529

El-Awaisi, A., Anderson, E., Barr, H., Wilby, K. J., Wilbur, K., \& Bainbridge, L. (2016). Passos importantes para a introdução da educação interprofissional na educação profissional de saúde. Journal of Taibah University Medical Sciences, 11 (6), 546-551. https://doi.org/10.1016/j.jtumed.2016.09.004.

Ellery, A. E. L. (2018). Interprofissionalidade. In R. B. Ceccim, D. Dallegrave, A. S. L. Amorim, V. de M. Portes, \& B. P. Amaral (Eds.), EnSiQlopédia das Residências em Saúde (pp. 146-150). Rede Unida. Recuperado de http://historico.redeunida.org.br/editora/biblioteca-digital/serie-vivencias-em-educacao-nasaude/ensiqlopedia-das-residencias-em-saude-pdf.

Ely, L. I e Toassi, R. F. C. (2018). Integração entre currículos na educação de profissionais da Saúde: A potência para educação interprofissional na graduação. Interface - Comunicação, Saúde, Educação, 22 (2), 1563-1575. https://doi.org/10.1590/1807-57622017.0658

Freire Filho, J. R, Costa, M. V da, Magnago, C., \& Forster, A. C. (2018). Atitudes em relação à colaboração interprofissional das equipes de Atenção Básica que participam do programa Mais Médicos (Mais Médicos). Revista Latino-Americana de Enfermagem, 26 (0). https://doi.org/10.1590/1518-8345.2731.3018.

Freire Filho, J. R., Silva, C. B. G., Costa, M. V. da, \& Forster, A. C. (2019). Educação Interprofissional nas políticas de reorientação da formação profissional em saúde no Brasil. Saúde em Debate, 43 (esp. 1), 86-96. https://doi.org/10.1590/0103-11042019s107.

Frenk, J., Chen, L., Bhutta, Z. A., Cohen, J., Crisp, N., Evans, T., Fineberg, H., Garcia, P., Ke, Y., Kelley, P., Kistnasamy, B., Meleis, A., Naylor, D., PablosMendez, A., Reddy, S., Scrimshaw, S., Sepulveda, J., Serwadda, D., \& Zurayk, H. (2010). Profissionais de saúde para um novo século: Transformando a educação para fortalecer os sistemas de saúde em um mundo interdependente. The Lancet, 376 (9756), 1923-1958. https://doi.org/10.1016/S01406736(10)61854-5.

Girard, M.-A. (2021). Educação interprofissional e políticas de prática colaborativa e direito: uma revisão internacional e questões reflexivas. Recursos Humanos para Saúde, 19 (1), 9. https://doi.org/10.1186/s12960-020-00549-w.

Guba, E. G., \& LINCOLN, Y. S. (2011). Avaliação de Quarta Geração. Editora da UNICAMP.

Lemos, A. B. (2019). Estágio docente no ensino superior: Relato de experiência no curso de Biblioteconomia da UFMG. Revista Docência do Ensino Superior, 9, 1-18. https://doi.org/10.35699/2237-5864.2019.12203.

Mello, C. de C. B., Alves, R. O., \& Lemos, S. M. A. (2014). Metodologias de ensino e formação na área de saúde: Revisão de literatura. Revista CEFAC, 16 (6), 2015-2028. https://doi.org/10.1590/1982-0216201416012.

Mielke, F. B., Olschowsky, A., Pinho, L. B. de, Wetzel, C., \& Kantorski, L. P. (2012). Avaliação qualitativa da relação de atores sociais com a loucura em um serviço substitutivo de saúde mental. Revista Brasileira de Enfermagem, 65 (3), 501-507. https://doi.org/10.1590/S0034-71672012000300016.

Moraes, B. A. (2016). Repensando a formação de profissionais de saúde à luz dos movimentos de mudança. Recuperado de https://repositorio.bc.ufg.br/tede/bitstream/tede/5555/5/Disserta\%C3\%A7\%C3\%A3o\%20-\%20Bibiana\%20Arantes\%20Moraes\%20-\%202016.pdf.

Moraes, B. A., Sousa, L. M. de, Menezes, I. H. C. F., Queiroz, M. G., Costa, N. M. da S. C., Guimarães, M. M., \& Ferreira, B. J. (2019). Avaliação do Impacto dos Programas de Reorientação Profissional em Cursos da Área da Saúde. Revista Brasileira de Educação Médica,43(2), 122129. https://doi.org/10.1590/1981-52712015v43n2rb20180162.

Moraes, M. M. dos S. de, Roner, M. N. B., Rocha, E. M. S., \& Maia, R. M. da C. S. (2019). Interdisciplinaridade e interprofissionalidade: Uma estratégia de ensino-aprendizagem na área de Parasitologia. Revista Docência do Ensino Superior, 9, 1-17. https://doi.org/10.35699/2237-5864.2019.13548

Organização Mundial da Saúde (2010). Marco para Ação em Educação Interprofissional e Prática Colaborativa. Genebra: Editora Freelance. http://www.paho.org/bra/images/stories/documentos/marco_para_acao.pdf.

Paim, J. S. (2018). Sistema Único de Saúde (Sus) aos 30 anos. Ciência \& Saúde Coletiva, 23 (6), 1723-1728. https://doi.org/10.1590/141381232018236.09172018.

Peduzzi, M. (2017). Educação interprofissional para o desenvolvimento de competências colaborativas em saúde. In R. F. C. Toassi (Ed.), Interprofissionalidade e formação na saúde: onde estamos? (pp. 49-67). Porto Alegre: Rede Unida. http://historico.redeunida.org.br/editora/bibliotecadigital/serie-vivencias-em-educacao-na-saude/vol-06-interprofissionalidade-e-formacao-na-saude-pdf. 
Research, Society and Development, v. 10, n. 9, e4310917618, 2021

(CC BY 4.0) | ISSN 2525-3409 | DOI: http://dx.doi.org/10.33448/rsd-v10i9.17618

Peduzzi, M., \& Agreli, H. F. (2018). Trabalho em equipe e prática colaborativa na Atenção Primária à Saúde. Interface - Comunicação, Saúde, Educação, 22 (supl 2), 1525-1534. https://doi.org/10.1590/1807-57622017.0827.

Poletto, P. R., \& Jurdi, A. P. S. (2018). Uma experiência de revisão das matrizes curriculares em um projeto pedagógico inovador: Caminhos para fortalecer a educação interprofissional em saúde. Interface - Comunicação, Saúde, Educação, 22 (supl 2), 1777-1786. https://doi.org/10.1590/1807-57622017.0819.

Reeves, S. (2016). Porque precisamos de educação interprofissional para melhorar a prestação de cuidados seguros e eficazes. Interface - Comunicação, Saúde, Educação, 20 (56), 185-197. https://doi.org/10.1590/1807-57622014.0092.

Reeves, S., Fletcher, S., Barr, H., Birch, I., Boet, S., Davies, N., McFadyen, A., Rivera, J., \& Kitto, S. (2016). Uma revisão sistemática BEME dos efeitos da educação interprofissional: Guia BEME No. 39. Medical Teacher, 38 (7), 656-668. https://doi.org/10.3109/0142159X.2016.1173663.

Reeves, S., Perrier, L., Goldman, J., Freeth, D., \& Zwarenstein, M. (2013). Educação interprofissional: efeitos na prática profissional e nos resultados da saúde. Cochrane Database of Systematic Reviews. https://doi.org/10.1002/14651858.CD002213.pub3.

Rossit, R. A. S., Freitas, M. A. de O., Batista, S. H. S. da S., \& Batista, N. A. (2018). Construção da identidade profissional na Educação Interprofissional em Saúde: Percepção de egressos. Interface - Comunicação, Saúde, Educação, 22 (suplemento 1), 1399-1410. https://doi.org/10.1590/1807-57622017.0184.

Rossit, R. A. S., Santos Junior, C. F. dos, Medeiros, N. M. H. de, Medeiros, L. M. O. P., Regis, C. G., \& Batista, S. H. S. da S. (2018). Grupo de pesquisa como espaço de aprendizagem em / sobre educação interprofissional (Eip): Narrativas em foco. Interface - Comunicação, Saúde, Educação, 22 (2), 15111523. https://doi.org/10.1590/1807-57622017.0674.

Santos, L. C. dos, Simonetti, J. P. e Cyrino, A. P. (2018). A educação interprofissional na graduação de Medicina e Enfermagem em prática na atenção primária à saúde: A perspectiva dos estudantes. Interface - Comunicação, Saúde, Educação, 22 (2), 1601-1611. https://doi.org/10.1590/1807-57622017.0507.

Stadick, J. L. (2020). A relação entre a educação interprofissional e as atitudes dos profissionais de saúde em relação ao trabalho em equipe e às competências colaborativas interprofissionais. Journal of Interprofessional Education \& Practice, 19, 100320. https://doi.org/10.1016/j.xjep.2020.100320

Stokes, C. K., Lysaght, C., Kim, Y., Lin, C.-C., Murphy, L., \& Radloff, J. C. (2020). Influência da comunicação interprofissional nas decisões de alta em alunos de pré-licenciamento da área de saúde. Journal of Interprofessional Education \& Practice, 19, 100329. https://doi.org/10.1016/j.xjep.2020.100329

Toassi, R. F. C., Olsson, T. O, Lewgoy, A. M. B., Bueno, D., \& Peduzzi, M. (2020). Ensino da graduação em cenários da atenção primária: Espaço para aprendizagem interprofissional. Trabalho, Educação e Saúde, 18 (2), e0026798. https://doi.org/10.1590/1981-7746-sol00267.

Wetzel, C. (2005). Avaliação de serviço em saúde mental: a construção de um processo participativo. http://www.teses.usp.br/teses/disponiveis/22/22131/tde$16052007-150813 /$ 